\title{
A New Approach for Reducing Embedding Noise in Multiple Bit Plane Steganography
}

\author{
Arijit Sur, Piyush Goel, and Jayanta Mukhopadhyay \\ Department of Computer Science and Engineering, \\ Indian Institute of Technology, Kharagpur \\ (arijits, piyush, jay)@cse.iitkgp.ernet.in
}

\begin{abstract}
In this paper, a new steganographic paradigm for digital images has been proposed. In order to reduce embedding noise we propose that information should be embedded in the scaled version of a grayscale value rather than directly in the grayscale value. This approach can be combined with any multiple bit plane embedding scheme to reduce the embedding noise. We also introduce a new steganographic scheme by combining the proposed approach with an existing multiple bitplane steganographic scheme and compare the performance of the combined scheme against the bare version of the existing steganographic scheme. Experimental results reveal that for same embedding rate a multiple bit plane embedding scheme combined with proposed approach adds less embedding noise and thus is less detectable against Wavelet Moment Analysis blind steganalytic attack than its bare version.
\end{abstract}

\section{Introduction}

The purpose of steganography is to hide the very presence of communication by embedding messages into innocent looking cover media like digital images. Thus the security or the undetectability of a steganographic algorithm is of foremost importance. LSB replacement is a well-known steganographic scheme where the Least Significant Bits of the cover image are replaced by the message bits. No significant visual distortion can be observed in a cover image when modifications are made in the LSB planes. But LSB replacement leads to a structural imbalance in the cover image which is exploited by the steganalytic attacks $[7,8]$ developed for the LSB replacement method.

In recent steganographic research $[2,3]$, the LSB method has been extended to multiple bit planes ( 2 or 3 planes). It should be noted that even LSB embedding upto 3 bitplanes does not cause significant visual distortion. Moreover, with respect to attacks based on the structural weakness of LSB method, higher bit plane LSB embedding is more secure than single bit plane LSB embedding [4]. On the other hand multiple bitplane embedding adds more noise in the cover image than single bit plane embedding. The amount of noise added during embedding depends not only the number of changes in the cover medium but also the amount of noise added per change. In the blind steganalysis paradigm it is often considered that statistical detectability of an embedding procedure is 
directly related to the total noise added during embedding. For a given medium, the steganographic algorithm which makes fewer embedding changes or adds less additive noise will be less detectable as compared to an algorithm which makes relatively more changes or adds higher additive noise [5].

The main objective of the proposed work is to suggest a new approach to data hiding which reduces the amount of embedding noise when applied jointly with some suitable multiple bit plane embedding scheme. The ability of the proposed approach to reduce noise lies in the observation that when a small number is embedded (added or subtracted) to a higher scaled version of a number(say carrier), then the embedding distortion in the carrier is less as compared to embedding the same small number into the unscaled version of the carrier. We combine this approach with an existing multiple bitplane embedding scheme and analyze the resulting noise and security of the combined scheme to validate the hypothesis.

The rest of the paper is organized as follows: in section 2, the proposed approach has been discussed, in section 3 we combine the proposed approach with an existing scheme to introduce a new steganographic scheme. In section 4, we give the detailed noise and security analysis of the combined steganographic scheme against an existing multiple bit plane scheme and finally conclusions are drawn in section 5 .

\section{Proposed Approach}

As discussed above, the embedding distortion is less when some modification is made to a scaled version of a number and the modified number is converted back to the original scale than making the same modification to the original version of the number. When a decimal number is converted to a lower base $p,(p<10)$, then the decimal coded value of the corresponding $p$ base number is always greater than the original decimal number. For example, consider a decimal number $x=156$, then the equivalent base 3 number $\left(x_{3}\right)$ will be $12210_{3}$. If we consider $x_{3}$ as a decimal value, it is twelve thousand two hundred and ten which is clearly greater than $x$. So we can say that $x$ is scaled in the $p$ base domain. Thus any change made to the $p$ base number is reduced when the changed $p$ base number is converted back to decimal base. We extend this concept to steganography in order to reduce the embedding noise when multiple bit planes are used for embedding. Any embedding operation can be modeled as a mathematical function (say $f$ ). Now rather than applying $f$ directly on a grayscale value ( $\operatorname{say} x$ ) of an image pixel for embedding, the grayvalue can be converted to a lower base number (say $x_{p}$ ) using Eq. 1.

$$
x_{p}=\sum_{i=0}^{k-1}\left[\bmod \left(\left\lfloor\frac{x}{p^{i}}\right\rfloor, p\right)\right] \times 10^{i}
$$

where $p<10$ and $x_{p}$ is a $k$ digit number. $x_{p}$ is considered as a decimal coded value and the embedding function $f$ is applied to $x_{p}$ producing $x_{p}^{\prime}$ as stego value in $p$ base domain i.e $x_{p}^{\prime}=f\left(x_{p}\right)$. Now the decimal equivalent of $x_{p}^{\prime}$ (say $x_{p}^{\prime \prime}$ ) can be computed by Eq. 2 . 


$$
x_{p}^{\prime \prime}=\sum_{i=0}^{k-1}\left[\bmod \left(\left\lfloor\frac{x_{p}^{\prime}}{10^{i}}\right\rfloor, 10\right)\right] \times p^{i}
$$

where $x_{p}^{\prime}$ is a $k$ digit number. The hypothesis is that $a b s\left(x-x_{p}^{\prime \prime}\right)<a b s(x-f(x))$ for a significant number of pixels of a grayscale image. It should be pointed out that embedding in the above fashion reduces noise for a percentage of grayscale values and not for all of the grayscale values. But it does lead to a significant reduction in the total embedding noise as explained in section 4 .

In the next section we combine this approach with an existing multiple bit planes embedding scheme, 3LSB embedding, to introduce a new steganographic scheme which adds less noise and thus is a secure steganographic scheme.

\section{Application Towards Steganography}

In this section we describe a new steganographic scheme which is a combination of the proposed base changing approach and 3LSB embedding scheme. In this scheme, the pixel values of grayscale cover image [range 0-255] are converted to a $p$ base system where $p<10$. This $p$ base number is treated as a decimal number and is modified to embed 3 message bits. After embedding if the modified pixel value remains a valid $p$ base number and the noise added in case of $p$ base embedding is less than normal decimal base embedding then embedding is allowed in $p$ base system and the stego $p$ base number is converted back to the corresponding decimal base. Otherwise embedding is done in the normal decimal base system. A shared secret parity function is used to convey the information whether embedding is in $p$ base or decimal base. For our experiments we use the $X O R$ operation as the parity function. We compare the proposed scheme with bare 3LSB scheme in order to determine the effectiveness of the proposed approach.

\subsection{Embedding Algorithm}

The algorithm for embedding $l$ message bits in a group of pixels is outlined below. The base $p$ is known apriori to both the encoder and the decoder.

- Divide the cover image into non overlapping groups of 2 pixels each. These groups can be formed using a pseudo-random sequence with a shared secret key.

- Consider $l$ message bits and compute their decimal equivalent $s$. The first pixel of the group (let it be denoted by $x$ ) is converted to a $p$ base system using Eq. 1 (let the $p$ base number be $x_{p}$ ). $x_{p}$ is treated as decimal coded number.

- The message symbol $s\left(0 \leq s<m\right.$ where $\left.m=2^{l}\right)$ is embedded in $x_{p}$ and also in $x$ using Eq. 3 which is the standard embedding function for $l$ bitplane LSB replacement method. Let the resulting values of $x_{p}$ and $x$ be $x_{p}^{\prime}$ and $x^{\prime}$ respectively. 


$$
x^{\prime}=\left(\left\lfloor\frac{x}{m}\right\rfloor \times m\right)+s
$$

where $x^{\prime}$ is the pixel value after embedding, $m=2^{l}$ and $s$ is the decimal equivalent of the $l$ message bits. In our case $l=3$ and $m=8$.

- $x_{p}^{\prime}$ may not be a valid $p$ base number or noise added due to $p$ base system may be greater than the noise added due to the decimal system. In such a case, the group is unsuitable for $p$ base embedding and message is embedded using normal decimal base i.e $x^{\prime}$ is considered as the stego value.

- After embedding, if the resultant stego value $\left(x_{p}\right)$ is a valid $p$ base number then its decimal equivalent $\left(x_{p}^{\prime \prime}\right)$ is calculated using Eq. 2 and is stored as stego pixel.

- Modify the LSB of second pixel of the group to embed the parity information using the $X O R$ function i.e. $X O R\left(l s b\left(x_{1}\right), l s b\left(x_{2}\right)\right)$ is equal to 1 and 0 for $p$ base embedding and decimal embedding respectively where $x_{1}$ and $x_{2}$ are the pixels of the group.

The extraction of the message sequence is a simple inverse process of the above algorithm as explained in the following example.

\subsection{Illustrative Example}

Consider two pixels $\left(a_{1}, a_{2}\right)$ having values 169 and 170 respectively and we want to embed the message sequence 110 (decimal value 6) using a base 4 system. $a_{1}$ is first changed to its base 4 representation, which is 2221 , using Eq. 1 . The message sequence is embedded in 2221 using Eq. 3 which gives 2222 as the embedded number. The corresponding base 10 equivalent of this number is 170 . Thus a noise of 1 is added which is much less as compared to noise added when embedding is done directly in 169 (a noise of 5 is added when embedding is done in base 10). Also we change $a_{2}$ to 171 to convey the parity information since $X O R(l s b(170), l s b(171))=1$. On the decoding end, we first check the parity information to find out whether decimal base embedding or $p$ - base embedding has been done. We see that $X O R(l s b(170), l s b(171))=1$ hence embedding has been done in $p$-base. So convert 170 to its base 4 representation i.e 2222 using Eq.1 and extract the decimal equivalent of the message sequence as 2222 modulo 8 (which equals 6 ). It can be checked that if value of $a_{1}$ and the message sequence was 164 and 101 respectively, then base 4 embedding would have added more noise as compared to decimal base embedding and hence embedding would have been done in decimal base system.

\section{Noise Analysis and Experimental Results}

\subsection{Noise Analysis}

The total noise due to above scheme comprises of the noise due to embedding in the $p$ base domain, the noise due to embedding in the decimal domain and 
the noise due to the parity information. The reduction in noise depends on the success rate of $p$ - base embedding. Since we dont have an exact statistical model wich accurately relates the success rate of $p$ base embedding and the reduction in noise, we provide empirical results observed from experiments on one hundered test images. On testing the algorithm on one hundered test images it was observed that on average, embedding in $p$ base system, for varying values of $\mathrm{p}$, was successfully accomplished for a significant number of pixels as shown in Table 1. The total noise is reduced in the new scheme as compared with bare 3LSB scheme as reflected in the scatter plot shown in Fig 1. $x$ axis and the $y$ axis depict the noise per pixel due to embedding by 3LSB and the proposed scheme respectively. Scatter plot was generated using $p=4$.

Table 1. Success Rate of $p$-base embedding for 100 images for different bases

\begin{tabular}{|c|c|c|c|c|c|c|c|}
\hline p base & 3 & 4 & 5 & 5 & 7 & 8 & 9 \\
\hline Success Rate & $24.45 \%$ & $22.99 \%$ & $29.23 \%$ & $20.77 \%$ & $37.72 \%$ & $30.60 \%$ & $39.62 \%$ \\
\hline
\end{tabular}

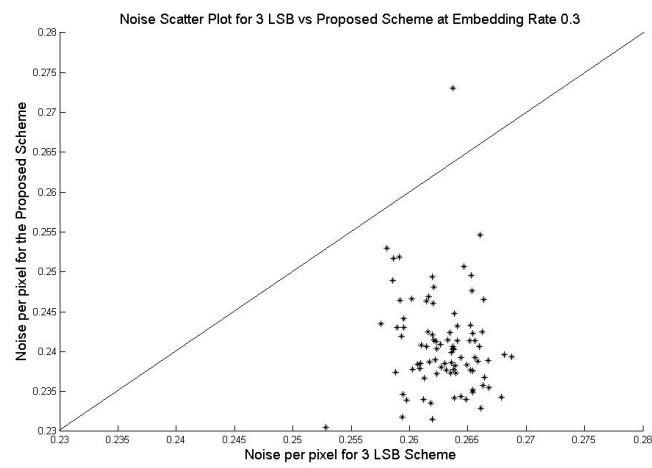

Fig. 1. Noise Scatter Plot Proposed Scheme vs 3LSB at Embedding Rate 0.30 bits per pixel. $x$ axis:Noise/pixel for 3LSB, $y$ axis:Noise/pixel for Proposed scheme.

We also compare the performance of the proposed scheme against 3LSB with respect to the embedding efficiency which is defined as the expected number of embedded message bits per one embedding change [11]. Table 2 gives a comparative analysis of 3LSB and proposed scheme for 2 values of pixel base $p(p=4$ and $p=8$ ) with varying embedding rates.

\subsection{Security Analysis}

A steganographic scheme is considered secure if given the full knowledge of the embedding algorithm, including the statistical properties of the source of the cover image, except the stego key, there are no artifacts in the stego image that 
Table 2. Average Additive Noise and Embedding Efficiency for 3LSB and proposed scheme

\begin{tabular}{|c|c|c|c|c|c|c|}
\hline Embedding & \multicolumn{2}{|c|}{ 3LSB } & Proposed Scheme Base = 4 & Proposed Scheme Base = 8 \\
\hline Rate & $\begin{array}{c}\text { Average } \\
\text { Noise }\end{array}$ & $\begin{array}{c}\text { Embedding } \\
\text { Efficiency }\end{array}$ & $\begin{array}{c}\text { Average } \\
\text { Noise }\end{array}$ & $\begin{array}{c}\text { Embedding } \\
\text { Efficiency }\end{array}$ & $\begin{array}{c}\text { Average } \\
\text { Noise }\end{array}$ & $\begin{array}{c}\text { Embedding } \\
\text { Efficiency }\end{array}$ \\
\hline 0.10 & 0.0874 & 1.1456 & 0.0800 & 1.2535 & 0.0736 & 1.3565 \\
\hline 0.20 & 0.1757 & 1.1386 & 0.1590 & 1.2541 & 0.1476 & 1.3578 \\
\hline 0.30 & 0.2630 & 1.1411 & 0.2386 & 1.2593 & 0.2213 & 1.3581 \\
\hline 0.40 & 0.3504 & 1.1417 & 0.3176 & 1.2609 & 0.2948 & 1.3584 \\
\hline 0.50 & 0.4376 & 1.1427 & 0.3964 & 1.2625 & 0.3684 & 1.3590 \\
\hline
\end{tabular}

can be detected by an attacker with a probability better than random guessing (Kerckhoffs principle) [1] . For evaluating the security of the proposed scheme, Wavelet Absolute Moment Steganalysis (WAM)[6] has been used as the steganlyzer. WAM is a blind steganalyser which classifies images as cover or stego based on a multi-dimensional feature space.The features for steganalysis are extracted from the noise component of the stego image in the wavelet domain. Assuming that the noise due to embedding is a stationary gaussian signal with a known variance, Wiener filter is used to extract noise component in the wavelet domain. All the features (statistical moments) are calculated as higher order moments of the noise residual in the wavelet domain. The total number of features for a grayscale image is $3 n_{\text {mom }}$ where $n_{\text {mom }}=9$. For classification, the 27 dimensional feature space is reduced to single dimension using Fisher Linear Discriminant (FLD) analysis. Then Linear Discriminant Analysis (LDA) classifier [10] is used to classify the projected points on the principal component axis and Receiver Operating Characteristic curves are plotted for evaluating the performance of the classifier on a steganographic algorithm.

\subsection{Experimental Results}

Two thousand uncompressed grayscale TIFF images of different sizes [9] were used for our experiments. The stego images were generated using the proposed steganograhic scheme for base 4 (i.e $p=4$ ) and bare 3LSB embedding scheme for embedding rates of 0.1 and $0.3 \mathrm{bits} /$ pixel. WAM classifier was trained with 1000 cover and 1000 stego images. ROC plots were generated for comparing the combined steganographic scheme with bare 3LSB embedding. It can be observed from the ROC plots shown in Fig. 2 and Fig. 3 that against WAM based steganalyzer the 3LSB scheme combined with proposed approach is less detectable than bare 3LSB embedding scheme. The proposed scheme generates more number of false positives than 3LSB and thus the classification procedure is closer to random guessing for proposed scheme. It should be mentioned that for higher embedding rates 3LSB scheme is detected fully by WAM due to large additive noise and hence the ROC plots for higher embedding rates have not been considered. 


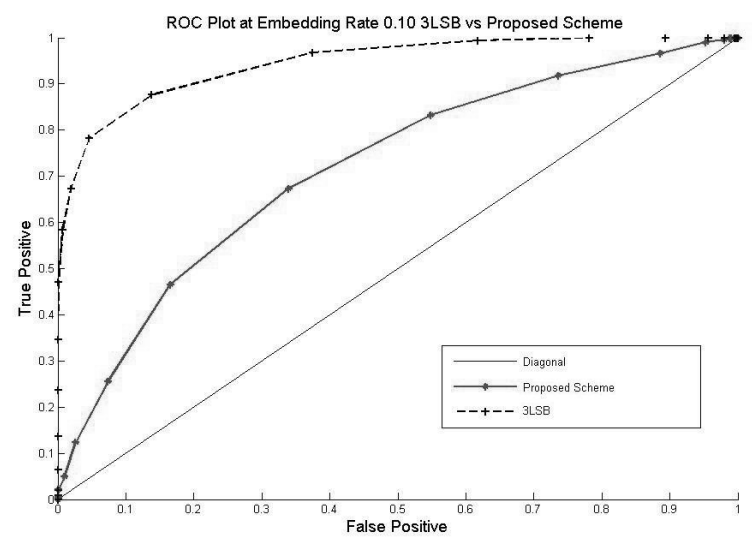

Fig. 2. ROC Plot:Proposed Scheme vs 3LSB Replacement at Embedding Rate 0.10 bits/pixel

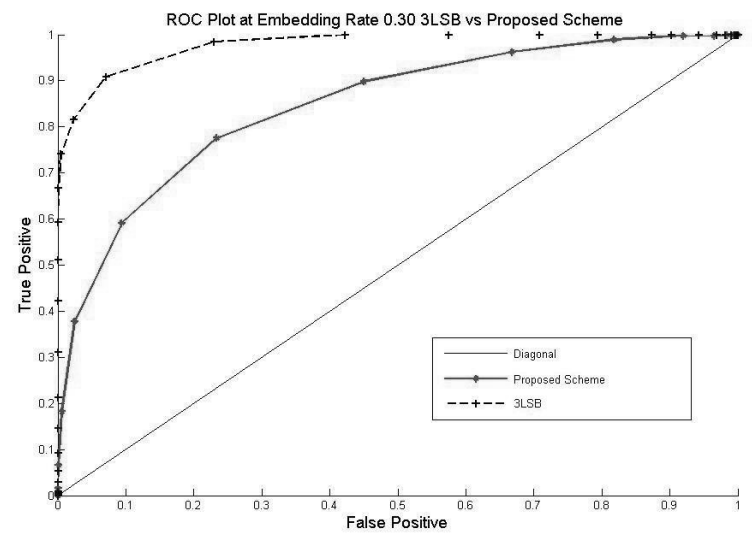

Fig. 3. ROC Plot:Proposed Scheme vs 3LSB Replacement at Embedding Rate 0.30 bits/pixel

\section{Conclusion}

In this paper, a new approach has been proposed which reduces the embedding noise when combined with a suitable multiple bit plane steganographic scheme. It has been shown empirically that when combined with 3LSB scheme the proposed method helps in reducing the embedding noise than bare 3LSB scheme and thus the merged scheme is statistically less detectable against WAM based steganalytic attack. Hence, the contribution of this paper is two-fold. Firstly a generalized base change method has been proposed which can be combined with different steganographic schemes. Secondly a secure steganographic 
algorithm has been introduced by combining the proposed approach with an existing steganographic scheme.

\section{References}

1. Katzenbeisser, S., Petitcolas, F.A.P.: Defining Security in Steganographic Systems. In: SPIE Security and Watermarking of Multimedia Contents IV. Electronic Imaging 2000, San Jose, CA, vol. 4675, pp. 50-56 (2002)

2. Zhang, X., Wang, S.: Steganography using multiple-base notational system and human vision sensitivity. Signal Processing Letters 12(1), 67-70 (2005)

3. Wu, D.-C., Tsai, W.-H.I.: A Steganographic method for images by pixel-value differencing. Pattern Recognit. Lett. 24, 1613-1626 (2003)

4. Ker, A.: Steganalysis of Embedding in Two Least-Significant Bits. IEEE Transaction on Information Forensics and Security 2(1) (March 2007)

5. Fridrich, J., Soukal, D.: Matrix Embedding for Large Payloads. In: Proc. of SPIE Electronic Imaging, Photonics West (January 2006)

6. Goljan, M., Fridrich, J., Holotyak, T.: New Blind Steganalysis and its Implications. In: Proc. SPIE Electronic Imaging, Photonics West (January 2006)

7. Dumitrescu, S., Wu, X., Wang, Z.: Detection of LSB steganography via sample pair analysis. In: Petitcolas, F.A.P. (ed.) IH 2002. LNCS, vol. 2578, pp. 355-372. Springer, Heidelberg (2003)

8. Fridrich, J., Goljan, M., Du, R.: Reliable detection of LSB steganography in color and grayscale images. In: Proc. ACM Workshop on Multimedia and Security, pp. 27-30 (2001)

9. Wang, J., Li, J., Wiederhold, G.: SIMPLicity: Semantics-sensitive integrated matching for picture LIbraries. IEEE Trans. Pattern Anal. Mach. Intell. 23(9), 947-963 (2001)

10. Fukunaga, K.: Introduction to Statistical Pattern Recognition. Academic Press, San Diego, California (1990)

11. Fridrich, J., Lisonek, P., Soukal, D.: On Steganographic Embedding Efficiency. In: 8th International Workshop Information Hiding, Alexandria, VA 